\title{
CROYANCES ET REPRÉSENTATIONS ENFANTINES DU RÊVE
}

\author{
Manuel Tostain et Joëlle Lebreuilly
}

P.U.F. | Enfance

2004/2 - Vol. 56

pages 149 à 162

\section{ISSN 0013-7545}

Article disponible en ligne à l'adresse:

http://www.cairn.info/revue-enfance-2004-2-page-149.htm

Pour citer cet article :

Tostain Manuel et Lebreuilly Joëlle, «Croyances et représentations enfantines du rêve », Enfance, 2004/2 Vol. 56, p. 149-162. DOI : 10.3917/enf.562.0149

Distribution électronique Cairn.info pour P.U.F..

(c) P.U.F.. Tous droits réservés pour tous pays.

La reproduction ou représentation de cet article, notamment par photocopie, n'est autorisée que dans les limites des conditions générales d'utilisation du site ou, le cas échéant, des conditions générales de la licence souscrite par votre établissement. Toute autre reproduction ou représentation, en tout ou partie, sous quelque forme et de quelque manière que ce soit, est interdite sauf accord préalable et écrit de l'éditeur, en dehors des cas prévus par la législation en vigueur en France. II est précisé que son stockage dans une base de données est également interdit. 


\title{
Croyances et représentations enfantines du rêve
}

\author{
Manuel Tostain et Joëlle Lebreuilly*
}

\section{RÉSUMÉ}

Une recherche a été entreprise auprès d'enfants âgés de 5 à 10 ans et d'étudiants afin de voir les évolutions des représentations et des croyances à propos du rêve. Quatre dimensions sont dégagées dans cette étude : la généralité du rêve, sa matérialité, la possibilité d'un contrôle personnel et le caractère surnaturel du rêve. Les résultats mettent en évidence que certaines croyances augmentent avec l'âge et varient selon le sexe des sujets. Cela nous amène à distinguer une double perspective, rationnelle et non rationnelle, dans la représentation du rêve, et on évoque la question du statut des dichotomies (réel/irréel, privé/public, interne/externe) habituellement utilisées pour traiter des phénomènes mentaux fictifs.

Mots clés: Enfants, Rêves, Représentations, Aspects rationnels et non rationnels.

\section{SUMMAR Y}

Beliefs and children's representations about dreams

A study has been carried out among 5-to-10-year-old children and students to determine evolutions about dream beliefs. Four dimensions are revealed: generality, materiality, personal control and surnatural characteristics of dreams. Results showed that some beliefs increased with age and are affected by sexual identity. We propose a double perspective, rational and non rational, and we discuss the status of dualisms mentioned usually in the study of fictional mental states (unreal-real, private-public, internal-external).

Key-words : Children, Dreams, Beliefs, Rational and Non Rational Perspective.

Ces vingt dernières années, dans le cadre du paradigme des théories de l'esprit, se sont développées de nombreuses recherches sur la représentation par l'enfant des contenus et des processus mentaux (Astington, 1993 ; Flavell, 1999 ; Flavell O’Donnell, 1999 ; Melot Nadel, 1999). Des avancées très

* UfR de Psychologie. Laboratoire de Psychologie cognitive et pathologique. Bureau SE 611, Université de Caen, 14032 Caen Cedex, Tél. : 02315665 93. E-mail : tostain@psycho.unicaen.fr.

ENFANCE, no 2/2004, p. 149 à 162 
importantes ont été faites, qui permettent d'avoir un tableau assez précis des évolutions enfantines au niveau de la compréhension des états mentaux tels que les intentions, les croyances ou les émotions d'autrui (Bradmetz Schneider, 1999). Néanmoins, le phénomène psychique particulier que constitue l'expérience onirique a reçu moins d'attention (Lilliard Flavell, 1992 ; Woolley, 1995).

À côté du livre princeps de Freud (1899) L'interprétation du rêve qui met l'accent sur les dynamiques inconscientes infantiles qui déterminent pour une part le contenu onirique, on sait que c'est à Piaget (1926) que l'on doit la première recherche systématique sur la représentation enfantine du rêve en tant qu'expérience mentale. Rappelons que, pour Piaget, l'enfant est initialement dans un état d'adualisme, d'indifférenciation soimonde qui entraîne deux tournures d'esprit principales - l'animisme qui consiste à projeter sur le monde des objets les caractéristiques de l'humain (vie, sensation, intentionnalité) - le réalisme qui se traduit par l'attribution aux phénomènes mentaux des propriétés du monde réel, comme par exemple la matérialité. Dans le cadre de ce réalisme, l'enfant verrait d'abord le rêve comme réel (c'est vrai), physique (on peut le toucher ou le voir), public (les autres peuvent le voir), et externe, tant au niveau de son origine (ça vient du dehors, apporté par la nuit ou envoyé par quelqu'un) que du lieu où il se déroule (à l'extérieur de la tête). Il faudrait attendre l'âge de 6,7 ans pour que l'enfant commence à percevoir la nature subjective du rêve, et la compréhension de sa dimension fictive (irréelle), non physique, interne et privée ne serait totalement atteinte que vers 11 ans. Des résultats assez proches ont été retrouvés par Laurendeau et Pinard (1962) puis par Kohlberg (1969). Dans cette optique piagétienne, les travaux de Foulkes (1993, 1999), repris notamment par Murray (1995), mettent en évidence un certain parallélisme entre, d'une part, le développement de la conscience de soi et d'autrui, l'évolution cognitive et, d'autre part, l'activité onirique enfantine. Ainsi, avec l'âge, les enfants rapportent de plus en plus fréquemment des rêves, les contenus s'enrichissent, deviennent plus imaginatifs et impliquent davantage le rêveur lui-même dans des interactions sociales qui, centrées initialement sur la famille, s'élargissent au groupe de pairs. Fait étonnant, on note que les cauchemars sont rares, les rêves ayant très généralement une tonalité plaisante.

Cependant, des recherches situées dans l'optique des théories de l'esprit (Estes, Wellman, Woolley, 1989; Wellman Estes, 1986) vont remettre en cause la généralité avancée par Piaget du réalisme enfantin. Les auteurs de ces études rappellent que l'on doit distinguer deux niveaux de réalismes: le réalisme ontologique qui renvoie à l'idée que les phénomènes mentaux sont des phénomènes physiques, le réalisme épistémologique qui consiste à considérer que tous les phénomènes mentaux sont forcément vrais. Concernant le premier réalisme, ils montrent que dès 3 ans les enfants sont capables de faire la distinction entre les entités physiques (un chien) et les entités mentales (la pensée d'un 
chien) ${ }^{1}$. Par contre jusqu'à 5 ans, les enfants présentent encore un certain réalisme épistémologique car ils ont quelques difficultés à différencier les pensées mentales renvoyant à la réalité et les états mentaux fictifs tels que l'imagination. Pour eux, les unes et les autres sont vrais. Appliqués à l'activité onirique, ces résultats suggèrent que les enfants sont capables de comprendre, à 3 ans, que le rêve est une entité non pas physique mais mentale et, à 5 ans, qu'elle est aussi fictive (que le rêve ne renvoie pas à une situation à laquelle le sujet a été réellement confronté même si le contenu peut être éventuellement réaliste). L'écart avec Piaget s'expliquerait, d'après ces auteurs, par l'utilisation par ce dernier de questions qui induisent une orientation matérialiste (par exemple «les rêves sont faits de quoi ?») et par une interprétation littérale de certaines réponses enfantines énoncées en fait de façon métaphorique (par exemple si l'enfant répond que le rêve se déroule à la montagne, dans l'esprit de l'enfant cela n'implique pas qu'il se réalise réellement à la montagne mais que tout se passe comme s'il se déroulait à la montagne). Néanmoins cette recherche de Wellman et Estes n'avait pas pour objectif spécifique la compréhension enfantine du rêve. C'est une étude de Woolley et Wellman, en 1992, qui va s'y attacher et mettre en évidence la précocité de cette compréhension. Ils confirmeront que, dès 3 ans, les enfants commencent à saisir la nature privée, non physique et interne du rêve, même si à cet âge ils sont encore nombreux à croire que plusieurs personnes peuvent avoir un rêve identique, partagé. Ces résultats ont été globalement confirmés par Kinoshita (1994) et Meyer et Shore (2001). Par la suite, Woolley et Boerger (2002) vont s'intéresser à la façon dont les enfants conçoivent l'origine des rêves et envisagent la possibilité de contrôler ce à quoi on rêve. Leurs recherches montrent que si, dès 5 ans, les enfants comprennent le rôle joué par les expériences passées, les sentiments personnels dans la détermination du rêve, ils surestiment pendant assez longtemps encore leur capacité à contrôler le contenu de leurs rêves. On peut dire que ce dernier résultat rejoint les travaux de Flavell et O'Donnel (1999) qui mettent en évidence que si les enfants ont une compréhension assez précoce de ce que sont les contenus mentaux (ce à quoi la personne pense), par contre, pour ce qui est des processus mentaux (comment les expériences mentales se réalisent), leur compréhension reste limitée et ils surestiment leurs possibilités. Par exemple, les enfants imaginent qu'on peut décider de penser strictement à rien pendant trois jours ou quand on est endormi profondément, que l'on peut percevoir, si l'on veut, ce qui se passe dans le monde extérieur. Pour rendre compte de ce phénomène, Flavell et O'Donnel rappellent que la compréhension des processus mentaux suppose d'être attentif à nos expériences mentales. Or la capacité

1. Des travaux complémentaires indiqueront que l'enfant dès 4 ans distingue la nature physique du cerveau et la dimension non physique de la pensée (Watson, Gelman, Wellman, 1998). 
d'introspection qui sous-tend justement cette attention est chez l'enfant d'âge préscolaire faible. Par ailleurs, dans leur recherche de 2002, Woolley et Boerger constatent un fait quelque peu surprenant, à savoir que les filles croient davantage au contrôle de leurs rêves que les garçons. Pour expliquer ce résultat, ces auteurs avancent que différentes études notent que les sujets féminins déclarent plus fréquemment que les sujets masculins rêver, et en parlent généralement plus. Les sujets féminins auraient ainsi une plus grande familiarité avec l'activité de rêver, ce qui développerait chez eux le sentiment de pouvoir contrôler leurs rêves. On pourrait ajouter que l'activité onirique relevant de la sphère émotionnelle qu'on considère, à tort ou à raison, comme davantage investie par les sujets féminins (Eagly Dickman, 1997), cet investissement les amènerait à être plus intéressés par cette idée de contrôle que les sujets masculins.

La plupart des travaux que l'on vient de citer analysent l'évolution avec l'âge de la représentation mentale du rêve dans une perspective généralement cognitive et universaliste. Il s'agit de voir à partir de quel âge l'enfant est capable de saisir certaines distinctions et l'on suppose d'une part, dans le cadre du développement cognitif, que certaines représentations erronées disparaissent avec l'âge et d'autre part que les enfants passent tous par les mêmes principales étapes. Par exemple, si on se réfère à Kohlberg (1969), il y a une séquence universelle invariable d'acquisition des différentes dimensions du rêve : les enfants comprennent d'abord la dimension irréelle du rêve, puis sa nature privée et enfin son caractère interne. Des auteurs soulignent cependant l'existence de variations culturelles à propos des théories de l'esprit des individus (Lillard, 1998). Et pour ce qui est du rêve, Shweder et Levine (1975) en étudiant une population particulière, l'ethnie nigérienne Hausa, mettent en évidence des séquences alternatives. Ainsi certains enfants Hausa par exemple, envisagent le rêve comme réel et public (visible par quelqu'un) et pourtant interne, ce qui n'est pas cohérent avec la séquence décrite par Kohlberg. Pour ces auteurs un tel écart avec les travaux de Kohlberg s'expliquerait en définitive par les croyances particulières de cette société africaine.

Tout cela suggère que si la perception des rêves s'inscrit dans un schéma évolutif cognitif, elle peut également renvoyer à des croyances culturelles qui ne dépendent pas uniquement de cette perspective cognitive et développementale (Kouassi, 1993 ; Levine, 1991). Á titre d'exemple encore, ces travaux anthropologiques relèvent que, dans certaines sociétés traditionnelles, les adultes, plus encore que les enfants, pensent que les rêves sont réels (par exemple renvoient à des événements qui vont réellement se produire dans l'avenir). On pourrait ajouter que ces croyances ne sont pas toujours culturellement homogènes, comme c'est le cas des croyances qui sont minoritaires à l'intérieur d'une culture donnée (Woolley, 1997 ; enquête CSA - Le Monde, 2003). Ainsi en est-il dans notre société occidentale des croyances parapsychologiques marginales sur la possibilité de communiquer avec les morts par le biais des rêves. Ici, on a affaire à des croyances que l'on peut 
qualifier de personnelles. Ajoutons de plus que bien souvent ces croyances ne sont, à proprement parler, ni vraies ni fausses car on ne peut leur faire subir le test de la vérification scientifique. Elles sont en ce sens non pas irrationnelles (résultant d'une faute logique) mais, dans la mesure où elles sont relatives à des systèmes de représentations du monde extrascientifiques, non rationnelles (Parot, 1995 ; Shweder, 1986 ; Sperber, 1982). Il est d'ailleurs parfois délicat de savoir ce qui relève ou non de la Science. Par exemple, si on reprend une question assez classique dans les études sur le rêve : "Peut-on voir les rêves de quelqu'un ", la réponse exacte semble évidemment non. Et pourtant, si on adopte un point de vue matérialiste sur la pensée, certains individus peuvent imaginer qu'avec les évolutions de l'imagerie cérébrale, un jour cette possibilité ne sera plus aussi insensée. Tout cela pour dire que, si l'évolution des représentations du rêve dépend à l'évidence de facteurs cognitifs, elle renvoie également à des logiques contextuelles et personnelles dont il est parfois difficile de prédire les lignes de forces développementales.

L'objectif de cet article sera de faire le point sur une population francophone des représentations enfantines du rêve en tant que phénomène mental, les études sur la question étant essentiellement anglo-saxonnes. En particulier, on cherchera à préciser quelles sont les croyances oniriques présentes chez l'enfant, ainsi que leurs évolutions avec l'âge et selon le sexe des sujets. On fera deux hypothèses. La première, que certaines croyances ne diminuent pas nécessairement avec l'âge, voire augmentent. La seconde, en se référant à l'expérience de Woolley et Boerger (2002), que les sujets féminins manifestent davantage certaines croyances que les sujets masculins.

MÉTHODE

Population : 175 sujets ont été interrogés : 133 enfants scolarisés en écoles primaires : 24 garçons et 24 filles de 5 ans (âge moyen $=5$ ans 4 mois ; étendue $=4$ ans 11 mois à 5 ans 10 mois), 20 garçons et 21 filles de 7 ans (âge moyen $=7$ ans 6 mois ; étendue $=7$ ans à 7 ans 11 mois), 24 garçons et 20 filles de 10 ans (âge moyen $=10$ ans 6 mois ; étendue $=10$ ans 2 mois à 10 ans 10 mois), 20 étudiants et 22 étudiantes de $1^{\text {re }}$ année de Sciences économiques (âge moyen $=19$ ans 2 mois; étendue 17 ans 11 mois à 23 ans 4 mois).

Procédure: Les enfants étaient interrogés dans leur école, individuellement et oralement, en dehors de la présence du professeur d'école, les adultes remplissant un questionnaire écrit. On introduisait la recherche par la phrase suivante : " La nuit, il arrive que l'on fasse des rêves. Je vais te poser quelques questions car je voudrais savoir ce que tu en penses. Il n'y a pas de bonnes ou de mauvaises réponses. » 


\section{Questionnaire : différents thèmes étaient abordés :}

Expérience du rêve : - Question 1 (Q1) : Est-ce que ça t'arrive de rêver la nuit? - Q2 : Est-ce que tu te souviens du dernier rêve que tu as fait? (on demandait au sujet de l'évoquer brièvement) - Q3 : Est-ce que tu fais des cauchemars ? - Q4 : Estce que tout le monde fait des rêves? - Q5 : Est-ce que les animaux rêvent? Aspect physique et public du rêve :- Q6 : Est-ce que tu penses que si quelqu'un rentre dans ta chambre pendant que tu rêves, il peut voir ton rêve, ce à quoi tu rêves ? - Q7 : Est-ce que tu penses qu'avec une machine spéciale, on peut voir les rêves (le contenu) que les gens font? Rêve et décision : - Q8 : Est-ce que tu peux décider de ce que tu vas rêver la nuit? - Q9 : Est-ce que pendant que tu rêves, tu peux décider de changer de rêve? (par exemple parce que celui que tu fais ne te plaît pas). Transmission du rêve : - Q10 : En te concentrant très fort, est-ce que tu peux envoyer un rêve à quelqu'un? - Q11: Et quelqu'un, en se concentrant très fort, est-ce qu'il peut t'envoyer un rêve? Caractère privé du rêve :- Q12 : Si tu dors dans la même chambre que ton meilleur copain (copine), est-ce que vous pouvez faire exactement le même rêve ? Caractère réel du rêve :-Q13 : Est-ce que tu penses que des fois, on rêve à des choses qui vont arriver en vrai ? (par exemple, tu rêves de quelqu'un que tu ne connais pas, que tu n'as jamais vu, et le lendemain, tu le rencontres en vrai) - Q14: Imagine que tu as fait une bêtise. Et que ta maman ne sache pas que c'est toi. Crois-tu que dans son rêve à elle, elle peut voir que c'est toi qui as fait cette bêtise ? Cauchemar et justice immanente :- Q15: Est-ce que tu crois que les cauchemars des fois, ils arrivent pour nous punir de choses pas gentilles qu'on a faites?

Codage: Les réponses oui étaient notées 1 , les réponses non 0.

\section{RÉSULTATS}

Traitements préalables: Dans un premier temps, nous avons procédé à une classification hiérarchique ascendante (mesure des distances euclidiennes, agrégation selon la méthode de Ward) afin de voir quelles étaient les questions qui présentaient une certaine proximité. L'analyse de l'arbre hiérarchique nous indique quatre groupes de variables (saut à $40 \%$ de Dmax) qui correspondent à différentes dimensions significatives du rêve. Un premier groupe est constitué par les questions 1 à 5 qui concernent la généralité du rêve (rêver la nuit, se souvenir de son dernier rêve, faire des cauchemars, est-ce que tout le monde rêve?, les animaux rêvent-ils?). Un deuxième groupe, représenté par les questions 6 et 7, renvoie à la matérialité du rêve (voir le rêve de quelqu'un dans sa chambre, voir les rêves avec une machine spéciale). Le troisième groupe (questions 8 à 10) touche à la croyance selon laquelle l'activité onirique résulte de processus volontaires, de décisions contrôlées par la personne qui rêve (possibilité de décider de ce que l'on va rêver, de changer si on le souhaite de rêve, d'envoyer un rêve à quelqu'un). Quant au dernier groupe (questions 11 à 15), on peut dire qu'il signale une conception clairement surnaturelle du rêve (notamment, idée 
que nos rêves sont envoyés par quelqu'un (Q11), notion de rêve prémonitoire (Q13) ou rétrospectif (Q14), de justice immanente (Q15)).

Pour approfondir l'analyse, ces quatre regroupements obtenus par la classification hiérarchique ont été transformés en indices. Pour ce faire, chaque sujet s'est vu attribuer une note par indice correspondant au nombre de réponses oui données à chacune des questions composant cet indice. Ensuite, afin de rendre comparable la lecture de ces indices, ceux-ci n'étant pas constitués d'un nombre équivalent de questions, nous avons transformé les scores sur 10. Ainsi, les scores par indice varient entre 0 (aucunes réponses oui aux questions constituant l'indice) et 10 (le sujet a répondu oui à toutes les questions de l'indice). Des analyses de variance ont ensuite été effectuées sur les indices. Nous ne présenterons que les résultats par âge pour les indices, car nous n'avons constaté aucuns effets simples du sexe, ni d'effets d'interaction âge $\times$ sexe des sujets. Au niveau de chacune des questions, nous avons également comparé les fréquences de réponses oui et non (calcul de Khi-Deux). Dans ce cas, on verra que se manifestent certaines différences selon le sexe de sujets.

Analyse des indices : Indice de généralité $(\mathrm{Q} 1$ à $\mathrm{Q} 5)$ : On note un effet de l'âge $(\mathrm{F}(3,167)=44,5, p<.00008)$, la perception de l'activité onirique et son " universalité » augmentant significativement avec l'âge. Ainsi, la note moyenne de l'indice, de 5,75 à 5 ans, atteint 9,44 chez les étudiants (voir tableau 1). Si on rentre dans le détail des questions qui constituent cet indice, on note que l'augmentation la plus nette concerne la question 5 sur la présence du rêve chez les animaux, le pourcentage de sujets répondant par l'affirmative passant de $4 \%$ à 5 ans à $80 \%$ chez les étudiants (sur les quatre groupes d'âge : Q5 : Khi-Deux (3) $=88,38, p<.00006$ ) (voir tableau 2). Une augmentation significative est également constatée pour la question 2 sur le souvenir de son dernier rêve $(\mathrm{Q} 2$ : Khi-Deux $(3)=42,15, p<.00002)$ et la question 4 ayant trait au fait que tout le monde rêve (Q4: KhiDeux (3) $=51,58, p<.00001)$. Toutefois, il y a une absence de l'effet de l'âge sur les questions qui renvoient au fait de rêver la nuit (Q1) ou de faire des cauchemars (Q3) (Khi-Deux non significatifs). L'explication réside ici dans un effet "plafond ", les réponses affirmatives étant déjà très fortes chez les enfants de 5 ans. Indice de matérialité (Q6 et Q7) : On relève un effet de l'âge $(\mathrm{F}(3,167)=3,11, p<.027)$, la croyance dans la possibilité de voir les rêves, globalement assez peu présente, diminuant avec l'âge (note de 3,12 à 5 ans, de 1,88 chez les étudiants). Plus précisément, cet effet de l'âge concerne la possibilité de voir le rêve de quelqu'un dans sa chambre qui diminue significativement (Q6 : Khi-Deux (3) = 20,46, $p<.001)$, mais pas l'idée de voir le rêve avec une machine (Q7 : Khi-Deux $(3)=6,531, p<.08$, ns). Cette dernière croyance est en effet présente à tous les âges, et à 10 ans elle est même majoritaire (59\% de réponses positives). Indice de contrôle personnel (Q8 à $\mathrm{Q} 10)$ : Il y a un effet de l'âge $(\mathrm{F}(3,167)=6,748, p<.0002)$, l'idée de contrôle diminuant avec l'âge (note de 4,17 à 5 ans, de 1,90 chez les étudiants). Cet effet de l'âge concerne uniquement l'idée de pouvoir envoyer un rêve à 
autrui (Q10: Khi-Deux $(3)=59,29, p<.00001)$ qui touche un enfant de 5 ans sur deux mais plus aucun étudiant. Par contre, on note une baisse mais non significative, pour l'idée de décider de ses rêves (Q8: KhiDeux (3) $=6,61, p<.0851)$ et aucune baisse pour la possibilité de changer de rêve $(\mathrm{Q} 9$ : Khi-Deux $(3)=4,96, p<.17)$ qui est à tous les âges partagé par environ un sujet sur trois. Indice de surnaturel $(\mathrm{Q} 11$ à Q15) : Pour ce dernier indice, on relève une absence de l'effet de l'âge $(\mathrm{F}(3,167)=1,96, p<.122)$. Cet indice masque cependant des variations significatives qui vont dans des sens opposés si on prend question par question. Ainsi, on note une baisse avec l'âge de la possibilité de recevoir un rêve (Q11 : Khi-Deux (3) = 10,69, $p<.0135)$, mais une augmentation de l'idée de faire des rêves prémonitoires (Q13 : Khi-Deux (3) = 8,574, $p<.035)$ ou de faire exactement le même rêve, spécialement entre 5 et 10 ans $(\mathrm{Q} 12$ : Khi-Deux $(3)=18,792, p<.00006)$. Enfin, on remarque un effet de contraste entre la croyance aux rêves « rétrospectif », qui est plus importante aux deux extrêmes de l'échelle d'âge (chez les 5 ans et les étudiants) (Q14 : Khi-Deux $(3)=12,823, p<.005)$, et la croyance dans la justice immanente, qui, elle, est majoritaire chez les sujets « centraux » (les 7 ans et 10 ans) (Q15: Khi-Deux $(3)=30,482, p<.00004)$. Corrélations entre indices: Le calcul des $\mathrm{R}$ de Bravais-Pearson met en évidence des liens faibles mais significatifs entre l'indice de matérialité et respectivement l'indice de contrôle personnel $(\mathrm{Rbp}=+.22, p<.003)$ et de surnaturel $(\mathrm{Rbp}=+.27, p<.001)$, et entre ces deux derniers indices $(\mathrm{Rbp}=+.25$, $p<.001)$.

TABleau 1. - Valeur moyenne des indices selon l'âge (note maxi 10)

\begin{tabular}{lllll}
\hline Âge & $\begin{array}{l}\text { Indice de } \\
\text { généralité }\end{array}$ & $\begin{array}{l}\text { Indice de } \\
\text { matérialité }\end{array}$ & $\begin{array}{l}\text { Indice } \\
\text { de contrôle } \\
\text { personnel }\end{array}$ & $\begin{array}{l}\text { Indice } \\
\text { de surna- } \\
\text { turel }\end{array}$ \\
\hline 5 ans & 5,75 & 3,12 & 4,17 & 3,29 \\
7 ans & 7,41 & 1,71 & 1,78 & 4,30 \\
10 ans & 8,40 & 2,87 & 1,87 & 4,30 \\
Étudiants & 9,44 & 1,88 & 1,90 & 3,45 \\
\hline
\end{tabular}

Analyse en fonction du sexe: Il n'y a aucun effet de cette variable au niveau des indices, et en ce qui concerne les questions prises isolément, cette variable n'agit que sur quatre des quinze questions. En l'occurrence, on constate que les filles déclarent davantage rêver la nuit (Q1: KhiDeux $(1)=8,479, p<.0003)$, décider du contenu de leurs rêves (Q8 : KhiDeux $(1)=5,836, p<.015)$ et recevoir des rêves (Q11: Khi-Deux $(1)=$ $11,206, p<.0008)$ tandis que les garçons avancent plus souvent que l'on peut voir les rêves avec une machine spéciale $(\mathrm{Q} 7$ : Khi-Deux $(1)=3,95$, $p<.05$ ) (voir tableau 3). 


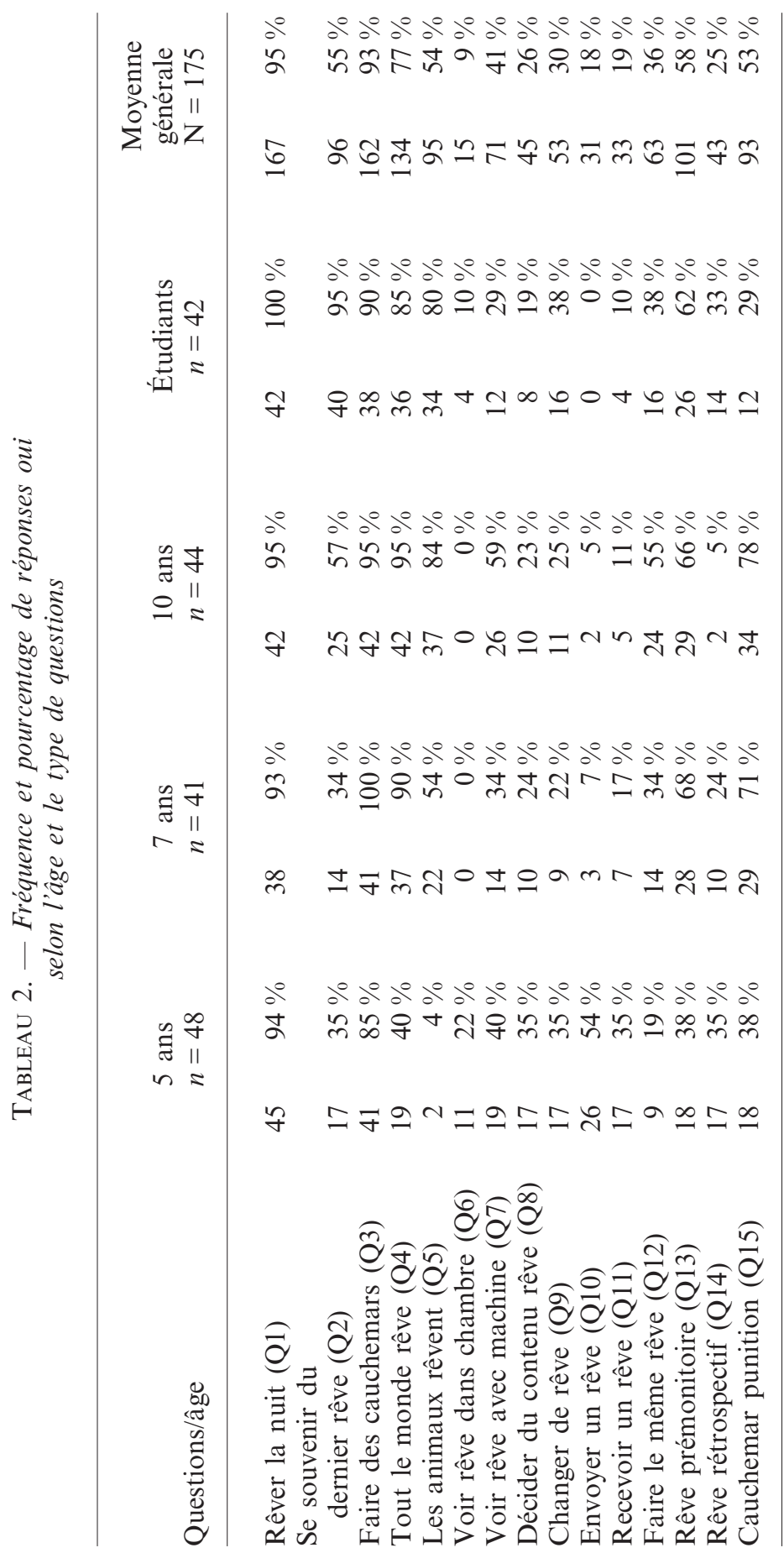


TABLEAU 3. - Fréquence et pourcentage de réponses oui selon le sexe

\begin{tabular}{|c|c|c|c|c|c|c|c|c|}
\hline \multirow{3}{*}{$\begin{array}{l}\begin{array}{l}\text { Sexe/ } \\
\text { questions }\end{array} \\
\text { Filles }(\mathrm{N}=87) \\
\text { Garçons }(\mathrm{N}=88)\end{array}$} & \multicolumn{2}{|c|}{$\begin{array}{l}\text { Rêver la } \\
\text { nuit (Q1) }\end{array}$} & \multicolumn{2}{|c|}{$\begin{array}{l}\text { Voir rêve } \\
\text { avec } \\
\text { machine } \\
\text { (Q7) }\end{array}$} & \multicolumn{2}{|c|}{$\begin{array}{l}\text { Décider } \\
\text { contenu } \\
\text { rêve }(\mathrm{Q} 8)\end{array}$} & \multicolumn{2}{|c|}{$\begin{array}{c}\text { Recevoir } \\
\text { rêve } \\
\text { (Q11) }\end{array}$} \\
\hline & 87 & 100 & 1 & 19 & 42 & $48 \%$ & 24 & $28 \%$ \\
\hline & 70 & $80 \%$ & 32 & 36 & 29 & $33 \%$ & 8 & 9 \\
\hline
\end{tabular}

DISCUSSION

Cette recherche met en évidence un certain nombre de choses. En premier lieu, la classification hiérarchique ascendante montre que quatre dimensions principales caractérisent dans notre étude la représentation du rêve (la généralité, la matérialité, le contrôle personnel et la dimension surnaturelle). On note aussi des liens cohérents entre les dimensions qui renvoient à des conceptions non rationnelles du rêve (cf. les corrélations entre les dimensions "contrôle personnel » et " conception surnaturelle »). Audelà de cette approche globale, une analyse détaillée révèle des aspects plus singuliers. Ainsi, si l'indice de généralité montre que la perception de l'activité onirique augmente avec l'âge, ce qui va dans le sens des travaux de Foulkes, on constate que cela est surtout lié à l'attribution, de plus en plus importante par les sujets quand ils s'élèvent en âge, de la possibilité pour les animaux de rêver. Sur la dimension matérielle du rêve, nos résultats montrent qu'il convient de distinguer deux types de matérialisme : Un matérialisme "primitif» qui consiste à voir le rêve comme quelque chose de physique, concret (cf. question " peut-on voir le rêve de quelqu'un dans sa chambre ») et qui diminue logiquement avec l'âge ; de l'autre un matérialisme qu'on pourrait qualifier de "périscientifique ». Là, il s'agit de l'idée que les rêves peuvent être vus par le biais d'une machine spéciale. Dans ce cas, ce matérialisme peut être rapproché des conceptions monistes (physicalistes) de la pensée, où le rêve est appréhendé sous la forme de train d'ondes électriques que des techniques d'imagerie cérébrale pourront un jour transformer en images mentales. C'est certainement pourquoi il n'y a pas ici d'effet de l'âge, car on peut dire qu'il ne s'agit pas d'une insuffisance cognitive mais d'un choix intuitif quant à la nature de l'activité mentale. Pour ce qui est de l'idée de contrôle personnel, il comporte également deux aspects. Un premier aspect ou niveau qui s'inscrit certainement dans le registre égocentrique enfantin d'une toute-puissance de la volonté et des désirs : C'est l'idée que l'on peut envoyer un rêve à quelqu'un et qui est spécifique des enfants de 5 ans. On a d'ailleurs un même effet de l'âge, mais moins marqué, à propos de l'idée de recevoir des rêves qui constitue en 
quelque sorte son symétrique. Pour ces deux thèmes, les enfants plus jeunes surestiment le pouvoir de la pensée, faute certainement d'être attentif à la manière dont se produisent les processus mentaux. Quant au second aspect de ce contrôle personnel, il concerne la possibilité de décider de ses rêves et d'en changer. On constate ici qu'il n'y a pas d'effet de l'âge, cette idée étant même assez fréquente chez les adultes, ce qui rejoint la recherche de Woolley et Boerger (2002). C'est au niveau de la dimension surnaturelle du rêve que l'on constate les résultats les plus étonnants. Ainsi, des conceptions parapsychologiques comme l'idée de rêve prémonitoire ou de rêve partagé augmentent avec l'âge. Quant à la notion de rêve rétrospectif (le rêve faisant référence à un événement réel du passé mais inconnu du rêveur), elle est très présente aux deux extrêmes de l'échelle d'âge. Pour les enfants de 5 ans, ce résultat doit être pris avec précaution car à cet âge la représentation du temps étant encore lacunaire (Crépault, 1994 ; Piaget, 1946), on peut se demander s'ils sont à même de comprendre véritablement ce que cette notion implique. On peut dire d'ailleurs la même chose de la présence à cet âge de la notion symétrique de rêve prémonitoire. Par contre, on peut supposer que la présence de ces deux conceptions chez les étudiants renvoie à une représentation assumée et clairement surnaturelle du rêve. Enfin, on note que l'idée de justice immanente (évoquée par la question du cauchemar comme punition), ne décroît pas de façon linéaire avec l'âge, les enfants de 7 ans et 10 ans étant les sujets qui y croient le plus, et ce dans leur grande majorité. La question de la justice immanente, sa signification (Piaget, 1932 ; Tostain, 1999) faisant débat, certains auteurs contestant sa présence chez l'enfant (Springer Ruckel, 1992), d'autres soulignant au contraire sa réalité (José, 1991), il est difficile d'interpréter ce résultat.

Concernant les différences selon le sexe des sujets, on note comme dans l'étude de Woolley et Boerger (2002), que les sujets féminins déclarent plus massivement rêver la nuit, avancent davantage l'idée de contrôle, mais aussi de pouvoir recevoir un rêve de quelqu'un, tandis que les garçons imaginent plus souvent que l'on peut voir les rêves avec une machine spéciale. Ici, même s'il l'on doit relativiser ces écarts (ils restent minoritaires), on peut dire que ces résultats renvoient peut-être à des centres d'intérêts différents. Socialement, les filles sont censées investir et exprimer davantage ce qui relève de la sphère affective, ce qui est le cas du rêve. Dès lors, c'est peutêtre ce plus grand sentiment de proximité avec l'activité onirique qui les disposerait à imaginer de telles possibilités psychologiques. Quant aux garçons, c'est peut-être l'intérêt plus prononcé pour la technique et ses virtualités (par exemple la Science-Fiction) qui les entraîne sur cette voie où une machine peut voir les rêves des individus.

Pour conclure, si les travaux actuels (Woolley, 1995) confirment que très tôt l'enfant est capable de faire la distinction entre les aspects irréels/réels, publics/privés, physiques/mentaux et externes/internes des activités mentales notamment dans le cas du rêve, nos résultats montrent qu'en parallèle persistent, voire se développent avec l'âge, des croyances qui rendent beaucoup 
moins ferme le statut de ces dichotomies. Ainsi, chez les enfants comme chez les adultes, certains sujets pensent que le rêve peut être prémonitoire (en ce sens réel), qu'on peut faire exactement le même rêve que quelqu'un d'autre (en ce sens public), qu'on peut le voir avec une machine (en ce sens physique) ou encore qu'il peut être envoyé par quelqu'un (en ce sens externe quant à son origine). Dès lors, pour comprendre ce type de résultats, il nous semble qu'il convient de distinguer à côté de la perspective rationnelle et développementale habituellement privilégiée dans les études actuelles sur le rêve, une perspective non rationnelle qui renvoie à des conceptions du monde personnelles et culturelles, non pas tant préscientifiques qu'extra-scientifiques. Cela dit, cette relative similarité parfois rencontrée entre les conceptions adultes et enfantines ne doit pas faire oublier évidemment la dimension développementale. En effet, si chez les adultes la nature non rationnelle de certaines croyances est généralement clairement perçue (Askevis-Leherpeux, 1988), chez les enfants, faute de connaissances scientifiques suffisantes, compte tenu de théories de l'esprit encore lacunaires, la frontière entre ce qui relève du rationnel et du non-rationnel n'est pas toujours bien délimitée. Pour autant cependant, tant chez les enfants que chez les adultes, ces croyances traduisent à notre avis une même tendance à vouloir aller au-delà des apparences et à donner du sens aux phénomènes humains (Harris, 1997 ; Johnson, 1997, Moscovici, 1992).

Pour la suite, il conviendrait justement de préciser quel statut les enfants accordent à ces différentes croyances (par exemple leur degré d'adhérence à ces croyances, comment ils les expliquent) et si des liens existent entre ces représentations du rêve et leurs représentations des phénomènes mentaux, la compréhension de ces derniers étant un élément important dans la conscience que nous avons de nous-même. Par ailleurs, il serait également intéressant de voir si ces croyances oniriques sont influencées par certaines conceptions personnelles, par exemple religieuses ou spirituelles.

\section{RÉFÉRENCES}

Askevis-Leherpeux, F. (1988). La superstition. Paris : Presses Universitaires de France. Astington, J. W. (1993). Comment les enfants découvrent la pensée. Paris : Retz.

Bradmetz, J., \& Schneider, R. (1999). La théorie de l'esprit dans la psychologie de l'enfant de 2 à 7 ans. Besançon: Presses Universitaires franc-comtoises.

Crépault, J. (1994). Notion de temps et raisonnement temporel. In R. Ghiglione, \& J. P. Richard (Eds), Cours de psychologie, Tome 3: Champs et méthodes (pp. 353-381). Paris: Dunod.

CSA - Le Monde - La Vie (2003). Les croyances des français. (Le Monde daté du mercredi 17 avril).

Eagly, A. H., \& Dickman, A. B. (1997). The accuracy of gender stereotypes : A dilemma for feminism. Revue internationale de psychologie sociale, 10, 2, 11-30.

Estes, D., Wellman, H. M., \& Woolley, J. D. (1989). Children's understanding of mental phenomena. In B. B. Wolman (Ed.), Handbook of developmental psychology (pp. 41-86). New York: Academic Press. 
Flavell, J. H. (1999). Cognitive development: Children's knowledge about the mind. Annual Review of Psychology, 50, 21-45.

Flavell, J. H., \& O'Donnell A. K. (1999). Le développement de savoirs intuitifs à propos des expériences mentales. Enfance, 3, 267-276.

Foulkes, D. (1993). Children's dreaming. In D. Foulkes, \& C. Cavallero (Eds), Dreaming as cognition (pp. 114-132). New York: Harvester.

Foulkes, D. (1999). Children's dreaming and the development of consciousness. Cambridge, MA : Harvard University Press.

Freud, S. (1899/2003). L'interprétation du rêve. Paris : Presses Universitaires de France.

Harris, P. L. (1997). The last of the magicians? Children, Scientists, and the invocation of hidden causal powers. Child Development, 68, 6, 1018-1020.

Johnson, C. N. (1997). Crazy children, fantastical theories, and the many uses of metaphysics. Child Development, 68, 6, 1024-1026.

José, P. E. (1991). Measurement issues in children's immanent justice judgments. MerrilPalmer Quarterly, 37, 4, 601-617.

Kinoshita, T. (1994). Young children's understanding of mental representations : Pretend and dream. Psychologia: An International Journal of Psychology in the Orient, 37, 1-6.

Kohlberg, L. (1969). Stage and sequence: The cognitive-developmental approach to socialization. In D. A. Goslin (Ed.), Handbook of socialization theory and research (pp. 347480). Chicago : Rand McNally.

Kouassi, K. (1993). Fonctions du rêve dans la société traditionnelle baoulé. Revue française de psychanalyse, 3, 883-887.

Laurendeau, M., \& Pinard, A. (1962). La pensée causale. Paris: Presses Universitaires de France.

Levine, J. B. (1991). The role of culture in the representation of conflict in dreams : A comparison of Bedouin, Irish, and Israeli children. Journal of Cross-Cultural Psychology, 22, $4,472-490$

Lillard, A. (1998). Ethnopsychologies : Cultural variations in theory of mind. Psychological Bulletin, 123, 1, 3-32.

Lillard, A. S., \& Flavell, J. H. (1992). Young children's understanding of different mental states. Developmental Psychology, 28, 4, 626-634.

Melot, A. M., \& Nadel, J. (1999). Comment l'esprit vient aux enfants. Enfance, 51, 3.

Meyer, S., \& Shore, C. (2001). Children's understanding of dreams as mental states. Dreaming- Journal of the Association for the Study of Dreams, 11, 4, 179-194.

Moscovici, S. (1992). La nouvelle pensée magique. Bulletin de psychologie, 405, 301-324.

Murray, J. B. (1995). Children's dreams. The Journal of Genetic Psychology, 156, 3, 303-312.

Parot, F. (1995). L'homme qui rêve. Paris : Presses Universitaires de France.

Piaget, J. (1926). La représentation du monde chez l'enfant. Paris: Presses Universitaires de France.

Piaget, J. (1932). le jugement moral chez l'enfant. Paris: Presses Universitaires de France.

Piaget, J. (1946). Le développement de la notion de temps chez l'enfant. Paris : Presses Universitaires de France.

Shweder, R. A. (1986). Divergent rationalities. In D. W. Fiske, \& R. A. Shweder (Eds), Metatheory in Social Science (pp. 163-196). Chicago and London: The University of Chicago Press.

Shweder, R. A., \& Levine, R. A. (1975). Dream concepts of hausa children : A critique of the «doctrine of invariant sequence» in cognitive development. Ethos, 3, 209-230.

Sperber, D. (1982). Les croyances apparemment irrationnelles. In Le savoir des anthropologues (pp. 49-86). Paris: Hermann.

Springer, K., \& Ruckel, J. (1992). Early beliefs about the cause of illness : Evidence against immanent justice. Cognitive Development, 7, 429-443.

Tostain, M. (1999). Psychologie, morale et culture: L'évolution de la morale de l'enfance à l'áge adulte. Grenoble: Presses Universitaires de Grenoble. 
Watson, J. K., Gelman, S. A., \& Wellman, H. M. (1998). Young children's understanding of the non-physical nature of thoughts and the physical nature of brain. British Journal of Developmental Psychology, 16, 321-335.

Wellman, H. M., \& Estes, D. (1986). Early understanding of mental entities : A reexamination of childhood realism. Child Development, 57, 910-923.

Woolley, J. D. (1995). The fictional mind: Young children's understanding of imagination, pretense, and dreams. Developmental Review, 15, 172-211.

Woolley, J. D. (1997). Thinking about fantasy: Are children fundamentally different thinkers and believers from adults? Child Development, 68, 6, 991-1011.

Woolley, J. D., \& Boerger, E. A. (2002). Development of beliefs about the origins and controllability of dreams. Developmental Psychology, 38, 1, 24-41.

Woolley, J. D., \& Wellman, H. M. (1992). Children's conceptions of dreams. Cognitive development, 7, 365-368. 